\title{
Multiplicative operations in the Steenrod algebra for Brown-Peterson cohomology
}

\author{
by
}

\author{
Michael S lack (Kalamazoo, Mich.)
}

\begin{abstract}
A family of multiplicative operations in the BP Steenrod algebra is defined which is related to the total Steenrod power operation from the mod $p$ Steenrod algebra. The main result of the paper links the BP versions of the total Steenrod power with the formal group approach to multiplicative BP operations by identifying the $p$-typical curves (power series) which correspond to these operations. Some relations are derived from this identification, and a short proof of the Hopf invariant one theorem is given as a sample computation.
\end{abstract}

1. Introduction. A classical tool used to study the Steenrod algebra is multiplicative operations derived from the reduced powers. In particular, one may define a total Steenrod power operation

$$
\mathcal{P}=\mathcal{P}^{0}+\mathcal{P}^{1}+\mathcal{P}^{2}+\mathcal{P}^{3}+\ldots,
$$

and use the fact that it is multiplicative in order to derive various formulas for the Steenrod algebra as a whole. This technique has the advantage that it reduces potentially large computations into nice generating function formulas, and it has been used in various forms.

When looking at Brown-Peterson cohomology, and its corresponding algebra of cohomology operations, there is a well-known technique for linking multiplicative operations to formal group laws [2]. Basically, the correspondence is that multiplicative operations coincide with homogeneous power series of the form

$$
f^{-1}(x)=\sum_{n \geq 0}^{F} c_{n} x^{p^{n}}
$$

where $c_{n} \in \mathrm{BP}^{*}$, and the sum is the formal sum determined by the formal group law for BP, which is universal among $p$-typical formal group laws. Such a power series is called a p-typical curve over $F$.

1991 Mathematics Subject Classification: Primary 55N20, 55N22, 55S25. 
It is the object of this paper to link these two important viewpoints. It is more or less well known that there are ways of defining an analogue of the operation $\mathcal{P}$ and other related operations for BP cohomology, and that these analogues will be multiplicative. There are, however, several possible choices for how to define them, and it is not obvious which choice is best. We show in this paper that one particular choice is most useful for linking the total power viewpoint with the formal group theory. We then demonstrate the link.

One way of viewing a $p$-typical curve is that it gives the action of the functional inverse of the corresponding $\mathrm{BP}$ operation on the Euler class $x \in \mathrm{BP}^{*}\left(\mathbb{C} P^{\infty}\right) \cong \mathrm{BP}^{*} \llbracket x \rrbracket$. Returning to $\bmod p$ cohomology, if we modify the definition of $\mathcal{P}$ so that it is a homogeneous operation

$$
\mathcal{P}=\mathcal{P}^{0}+u^{p-1} \mathcal{P}^{1}+u^{2(p-1)} \mathcal{P}^{2}+u^{3(p-1)} \mathcal{P}^{3}+\ldots,
$$

where $u$ is an indeterminate of degree -2 , then we may consider the action of $\mathcal{P}$ on the Euler class $x \in H^{*}\left(\mathbb{C} P^{\infty} ; \mathbb{Z} / p\right)$. The result is

$$
\mathcal{P}(x)=x+u^{p-1} x^{p} .
$$

If we let $\chi$ denote the antiautomorphism of the Steenrod algebra [9], so that

$$
\chi(\mathcal{P})(\mathcal{P}(x))=x,
$$

then a routine calculation yields

$$
\chi(\mathcal{P})(x)=x-u^{p-1} x^{p}+u^{p^{2}-1} x^{p^{2}}-u^{p^{3}-1} x^{p^{3}}+\ldots
$$

Now Corollary 3.3 of Theorem 1.1 says that for each $m$ there is a way of lifting $\mathcal{P}^{m}$ to a $\mathrm{BP}$ operation (we use the same name) so that the $p$-typical curve corresponding to $\mathcal{P}$ is the same as the one from (6), except with ordinary sums replaced by formal sums.

This phenomenon generalizes to other operations as well. There are several multiplicative operations related to $\mathcal{P}$ which can be formed from the Steenrod algebra. We describe a particular family of operations here. Let $\delta=\left(\delta_{1}, \delta_{2}, \delta_{3}, \ldots\right)$ denote a sequence of zeros and ones indexed by the natural numbers, where only finitely many of the $\delta_{n}$ are not zero. Let $H_{u}$ represent periodic mod $p$ cohomology, where $H_{u}^{*}=H^{*}\left[u, u^{-1}\right]$ and $|u|=-2$. Define the multiplicative operation $M_{\delta} \in H_{u}^{*} H$ by

$$
M_{\delta}=\sum_{\delta E} u^{|\delta E|} \mathcal{P}^{\delta E}
$$

where the sum ranges over all exponential sequences of the form

$$
\delta E=\left(\delta_{1} e_{1}, \delta_{2} e_{2}, \delta_{3} e_{3}, \ldots\right) .
$$

In other words, $M_{\delta}$ is the sum of all the elements of the Milnor basis [9] which have zeros in all of the same slots as $\delta$. We may also define $M_{\delta} \in \mathrm{BP}_{u}^{*} \mathrm{BP}$, 
where $\mathrm{BP}_{u}$ represents the periodic version of $\mathrm{BP}$, and Theorem 1.1 identifies the corresponding $p$-typical curve.

THEOREM 1.1. The multiplicative operation $M_{\delta}$ corresponds to the $p$ typical curve

$$
f_{\delta}^{-1}(x)=\sum_{n \geq 0}^{F}\left[\chi\left(\delta_{n}\right)\right]_{F} u^{p^{n}-1} x^{p^{n}},
$$

where $\chi\left(\delta_{n}\right)$ is determined by the recursive formula

$$
\chi\left(\delta_{0}\right)=1, \quad \chi\left(\delta_{n}\right)=-\sum_{k=0}^{n-1} \delta_{n-k} \chi\left(\delta_{k}\right) \quad(n>0),
$$

and its effect on the coefficient ring is given by

$$
M_{\delta}\left(\lambda_{k}\right)=\sum_{j=0}^{k} \chi\left(\delta_{k-j}\right) \lambda_{j} u^{p^{k}-p^{j}} .
$$

REMARK. The formula for $\chi\left(\delta_{n}\right)$ may also be obtained as the coefficient of $u^{p^{n}-1} x^{p^{n}}$ in the power series $f_{\delta}^{-1}(x) \in H_{u}^{*}\left(\mathbb{C} P^{\infty}\right)$, where

$$
f_{\delta}(x)=x+\sum_{n \geq 1} \delta_{n} u^{p^{n}-1} x^{p^{n}}=M_{\delta}(x) .
$$

Thus the $p$-typical curve corresponding to the operation $M_{\delta} \in \mathrm{BP}_{u}^{*} \mathrm{BP}$ is the one that looks exactly like the series that results by applying $\chi\left(M_{\delta}\right)$ to the Euler class in mod $p$ cohomology, and then replacing ordinary sums with formal sums.

The coefficients $\lambda_{k}$ are defined in Section 2 .

We are also interested in studying the subalgebra $P \subset \mathrm{BP}^{*} \mathrm{BP}$ generated by the primitive elements $\mathcal{P}^{\Delta_{k}}, k \geq 1$. Here $\Delta_{k}$ is the exponential sequence which consists of all zeros except for a one in the $k$ th place. In $H^{*} H$, the subalgebra $P$ is a truncated polynomial algebra generated by the $\mathcal{P}^{\Delta_{k}}$, where each $\mathcal{P}^{\Delta_{k}}$ has height $p$. The situation in $\mathrm{BP}^{*} \mathrm{BP}$ differs somewhat; $P$ is not a truncated polynomial algebra. The main result is given in Theorem 1.2.

Theorem 1.2. The subalgebra $P \subset \mathrm{BP}^{*} \mathrm{BP}$ generated by the primitive elements is a polynomial subalgebra. Furthermore, the rationalization $P \otimes \mathbb{Q}$ is isomorphic as an algebra to $\mathrm{BP}^{*} \mathrm{BP} \otimes \mathbb{Q}$.

The inclusion of the subalgebra $P \subset \mathrm{BP}^{*} \mathrm{BP}$ is not as well understood as one might like. For example, Lemma 3.1 suggests that, as a left $\mathrm{BP}^{*}$ module, $P$ might be isomorphic to the submodule of $\mathrm{BP}^{*} \mathrm{BP}$ generated by the elements $E ! \mathcal{P}^{E}$, where $E$ runs over all exponential sequences ( $E$ ! is defined by (26)). This is probably false, but the author does not know of a 
proof in either direction. A nice closed description of $P$ as a $\mathrm{BP}^{*}$-submodule of $\mathrm{BP}^{*} \mathrm{BP}$ would be interesting to find.

One of the original discoveries that led to this paper was a family of formulas that relate the elements of the Milnor basis for $\mathrm{BP}^{*} \mathrm{BP}$ to the elements of $P$. These formulas are useful for doing calculations, since the generators of $P$ are derivations, and rationally they generate all of $\mathrm{BP}^{*} \mathrm{BP}$. These formulas are summarized in Theorem 1.3.

THEOREM 1.3. The elements of $P$ are related to the elements of the Milnor basis for $\mathrm{BP}^{*} \mathrm{BP}$ by the formulas

$$
M_{\delta}=\operatorname{Exp}\left(-\sum_{0 \leq j<k} \chi\left(\delta_{k-j}\right) \lambda_{j} u^{p^{k}-p^{j}} \mathcal{P}^{\Delta_{k}}\right) .
$$

WARNING. The Exp above is not the exponential series for BP. It is the usual exponential series from elementary calculus

$$
\operatorname{Exp}(x)=\sum_{n \geq 0} \frac{x^{n}}{n !} .
$$

We distinguish it from the $\mathrm{BP}$ exponential, exp, by capitalizing the $\mathrm{E}$ in Exp.

WARnIng. One must take care in the sum from Theorem 1.3. Different orderings of the sum will give distinct operations. The sum above should be expanded so that the ordering from left to right is decreasing with respect to $k$.

The layout of this paper is as follows. In Section 2, the necessary background and terminology for Brown-Peterson cohomology is reviewed. Then the operations $\mathcal{P}^{E} \in \mathrm{BP}^{*} \mathrm{BP}$ are defined, and their relationship with the logarithm is explained. We then give a bit of terminology for dealing with exponential sequences. In Section 3, the main theorems on multiplicative operations are proved, and in Section 4, we use Theorem 1.3 to give a short proof of the Hopf invariant one theorem.

2. BP cohomology and the Steenrod algebra. Recall that BrownPeterson cohomology is determined by its coefficient ring $\mathrm{BP}^{*}=\mathrm{BP}^{*}(p t) \cong$ $\mathbb{Z}_{(p)}\left[v_{1}, v_{2}, v_{3}, \ldots\right]$, where $\left|v_{i}\right|=-2\left(p^{i}-1\right)$. A standard, and very useful, way of looking at $\mathrm{BP}^{-*}=\mathrm{BP}_{*}=\pi_{*}(\mathrm{BP})$ is to view it as a subset of $H_{*}\left(\mathrm{BP} ; \mathbb{Z}_{(p)}\right) \cong \mathbb{Z}_{(p)}\left[\lambda_{1}, \lambda_{2}, \lambda_{3}, \ldots\right]$ via the Hurewicz homomorphism. Sometimes the generators $\lambda_{i}$ are denoted by $m_{i}$ or $m_{p^{i}-1}$ in the literature. We use the notation from Ravenel's book [10]. The $\lambda_{i}$ are in the same degree as the $v_{i}$, but are not contained in $\mathrm{BP}^{*}$. However, they can be considered as elements of $\mathrm{BP}^{*} \otimes \mathbb{Q}$, and when there is no torsion (which is all of the 
time when we are dealing with universal examples), no information is lost in using the $\lambda_{i}$ instead of the $v_{i}$ in our various formulas.

For the purposes of this paper, we also wish to use the version of BP theory which is periodic; we distinguish this theory from BP by using the notation $\mathrm{BP}_{u}$. The coefficients are $\mathrm{BP}_{u}^{*}=\mathrm{BP}^{*}\left[u, u^{-1}\right]$, with $|u|=-2$. The periodic theory can be constructed using the Landweber exact functor theorem from [7], as follows. Using the map

$$
\mathrm{BP}^{*} \rightarrow \mathrm{BP}_{u}^{*}
$$

which sends $v_{i}$ to $v_{i}$, define a functor from spectra to $\mathrm{BP}_{u *}$-modules by

$$
\mathrm{BP}_{u *}(E)=\mathrm{BP}_{u *} \otimes_{\mathrm{BP}_{*}} \mathrm{BP}_{*}(E) .
$$

The $\mathrm{BP}^{*}$-module $\mathrm{BP}_{u}^{*}$ is Landweber exact, so this functor is a (multiplicative) homology theory. There is an isomorphism (of ungraded rings) $\mathrm{BP}^{*} \cong \mathrm{BP}_{u}^{0}$ which is induced by the map sending $v_{i}$ to $u_{i}=u^{1-p^{i}} v_{i}$.

If $H$ is the mod $p$ Eilenberg-MacLane spectrum, then the Hopf algebra $H^{*} H$ is called the Steenrod algebra. Many of its properties are well known; we assume the standard facts are known to the reader. A standard reference is [9]. We call $\mathrm{BP}^{*} \mathrm{BP}$ the Steenrod algebra for Brown-Peterson cohomology. The approach taken in this paper for studying $\mathrm{BP}^{*} \mathrm{BP}$ is motivated by the approach of [9] for the mod $p$ Steenrod algebra, using the dual $\mathrm{BP}_{*} \mathrm{BP}$.

Recall that $\mathrm{BP}_{*} \mathrm{BP}$ is a polynomial algebra

$$
\mathrm{BP}_{*} \mathrm{BP} \cong \mathrm{BP}_{*}\left[t_{1}, t_{2}, t_{3}, \ldots\right],
$$

where $\left|t_{i}\right|=2\left(p^{i}-1\right)$. The $t_{i}$ are standard generators, and are defined inductively by the right unit formula

$$
\eta_{\mathrm{R}}\left(\lambda_{k}\right)=\sum_{i=0}^{k} \lambda_{i} t_{k-i}^{p^{i}} .
$$

Let $\chi: \mathrm{BP}_{*} \mathrm{BP} \rightarrow \mathrm{BP}_{*} \mathrm{BP}$ be the antiautomorphism (induced by the twist map $\mathrm{BP} \wedge \mathrm{BP} \rightarrow \mathrm{BP} \wedge \mathrm{BP})$, and let $h_{i}=\chi\left(t_{i}\right)$. Then a simple inductive argument shows that

$$
\mathrm{BP}_{*} \mathrm{BP} \cong \mathrm{BP}_{*}\left[h_{1}, h_{2}, h_{3}, \ldots\right],
$$

where $\left|h_{i}\right|=2\left(p^{i}-1\right)$. The $h_{i}$ are used in [3].

The monomials in the $h_{i}$ form a $\mathrm{BP}_{*}$-module basis for $\mathrm{BP}_{*} \mathrm{BP}$. Following the method in [9], one may form the dual basis: for each exponential sequence $E=\left(e_{1}, e_{2}, e_{3}, \ldots\right)$, let $\mathcal{P}^{E} \in \mathrm{BP}^{*} \mathrm{BP}$ be the element $\mathrm{BP}_{*}$-dual to the monomial $h^{E}=h_{1}^{e_{1}} h_{2}^{e_{2}} h_{3}^{e_{3}} \ldots$ Then as $E$ runs through all exponential sequences, the $\mathcal{P}^{E}$ form a basis for $\mathrm{BP}^{*} \mathrm{BP}$ as a left $\mathrm{BP}^{*}$-module. The $\mathcal{P}^{E}$ defined above are $\mathrm{BP}^{*} \mathrm{BP}$ lifts of the usual $\mathcal{P}^{E} \in H^{*} H$. This is taken as well known; a proof can be found in Kane's paper [6]. We could have chosen a different set of generators than the $h_{i}$ to define the $\mathcal{P}^{E}$, but as mentioned 
in the introduction this particular choice is the one for which Theorem 1.1 holds.

The multiplicative operations $M_{\delta}$ of Theorem 1.1 are actually elements of $\mathrm{BP}_{u}^{*} \mathrm{BP}$ rather than $\mathrm{BP}^{*} \mathrm{BP}$. This is not a problem, however, as a $p$-typical curve $f(x) \in \mathrm{BP}_{u}^{*}\left(\mathbb{C} P^{\infty}\right)$ is represented by a map

$$
\mathbb{C} P^{\infty} \rightarrow \mathrm{BP}_{u}
$$

Hence the universality of BP for $p$-typical formal group laws will have the corresponding multiplicative operation represented by a ring spectrum map

$$
\mathrm{BP} \rightarrow \mathrm{BP}_{u}
$$

In [4], Hubbuck defines operations, via the Chern character in K-theory, which are integral liftings of Steenrod operations. The connection between Hubbuck's operations and BP operations is described in Kane's paper [5]; we briefly explain the connection here. Define a multiplicative operation $\Lambda \in \mathrm{BP}^{*} \mathrm{BP} \otimes \mathbb{Q}$ by the formula

$$
\Lambda=\sum_{E} \lambda^{E} \mathcal{P}^{E}
$$

where $\lambda^{E}=\lambda_{1}^{e_{1}} \lambda_{2}^{e_{2}} \lambda_{3}^{e_{3}} \ldots$ (This operation is not an element of $\mathrm{BP}^{*} \mathrm{BP}$, since the $\lambda_{k}$ are not contained in $\left.\mathrm{BP}^{*}\right)$. The operation $\Lambda$ is characterized by its being the unique multiplicative operation for which $\Lambda\left(\lambda_{k}\right)=0$ if $k>0$. The proof of this follows from the right unit formula (30) in Lemma 3.2. It also depends on the fact that a multiplicative operation is determined by its values on the $\lambda_{k}$. In fact, the corresponding $p$-typical curve is $f_{\Lambda}^{-1}(x)=$ $\exp (\operatorname{mog} x)$, where

$$
\operatorname{mog} x=\sum_{k \geq 0} \Lambda\left(\lambda_{k}\right) x^{p^{k}}=x .
$$

Hence $f_{\Lambda}^{-1}(x)=\exp x$.

One could attempt to define the $\mathcal{P}^{E}$ from $\Lambda$ by starting with $f_{\Lambda}^{-1}(x)=$ $\exp x$. This is essentially what Hubbuck does in [4], except using K-theory instead of BP. There is a problem with this approach, however. The $\mathcal{P}^{E}$ are not uniquely defined by this process, because the $\lambda^{E}$ have negative degree. One can define the $\mathcal{P}^{E}$ uniquely only modulo higher filtration, otherwise some choices must be made. In his situation, Hubbuck chooses arbitrarily, so he is only able to prove certain properties modulo higher filtration. In our case, we choose to define the $\mathcal{P}^{E}$ in a very particular way, and that has the advantage that our formulas are exact.

There are certain conventions regarding exponential sequences that are used in this paper which keep notation simple. Recall from [9] that an $e x$ ponential sequence $E$ is a sequence of non-negative numbers $\left(e_{1}, e_{2}, e_{3}, \ldots\right)$ only finitely many of which are not zero. The degree of $E$, denoted by $|E|$, 
is equal to the sum

$$
|E|=\sum_{n \geq 1} e_{n}\left(p^{n}-1\right),
$$

the excess, $e(E)$, is given by

$$
e(E)=\sum_{n \geq 1} e_{n},
$$

and the symbol $E$ ! refers to the product

$$
E !=e_{1} ! e_{2} ! e_{3} ! \ldots
$$

A standard notation that we use is $\Delta_{k}$ for the sequence which consists of all zeros except for a one in the $k$ th space. There are also several places where there is some indexed set of symbols, for example the $\lambda_{k}$, and we refer to $E$ as an exponent, as in $\lambda^{E}$. In this case the notation should be interpreted so that the entries of $E$ are the various exponents in a product expansion, as in

$$
\lambda^{E}=\lambda_{1}^{e_{1}} \lambda_{2}^{e_{2}} \lambda_{3}^{e_{3}} \cdots
$$

3. Multiplicative operations. In this section the main results of the paper are proved. First, Lemma 3.1 gives a formula for the action of $P$ on the rational coefficient ring for BP. Theorem 1.2 is a direct consequence of Lemma 3.1. In order to prove Lemma 3.1, a second technical lemma is needed which describes the right unit homomorphism in a particularly useful way.

We then prove the main result of the paper, Theorem 1.1, which identifies the $p$-typical curves associated with the multiplicative operations $M_{\delta}$. The beauty of Theorem 1.1 is that it is a clear generalization of what is known for the $\bmod p$ Steenrod algebra. In fact, one may characterize Theorem 1.1 as taking what is known for the $M_{\delta}$ in the $\bmod p$ Steenrod algebra, and replacing ordinary sums with formal sums.

The identification of the curves associated with the $M_{\delta}$ allows one to easily compute the action of $M_{\delta}$ on the coefficients. Since the $M_{\delta}$ are completely determined by this action, we may use these formulas, together with Lemma 3.1, to prove Theorem 1.3, which relates the $M_{\delta}$ to certain rational multiplicative operations constructed from the subalgebra $P$.

\section{LEMMA 3.1. We have}

$$
\left(\mathcal{P}^{\Delta}\right)^{E} \lambda^{F}=(-1)^{e(E)}(F ! /(F-E) !) \lambda^{F-E} .
$$

Hence the elements

$$
\left(\mathcal{P}^{\Delta}\right)^{E} / E !
$$


form an additive basis for $\mathrm{BP}^{*} \mathrm{BP} \otimes \mathbb{Q}$ as $E$ runs over all exponential sequences.

The proof of this lemma is obtained from the right unit homomorphism $\eta_{\mathrm{R}}$. Before proceeding, another lemma is needed.

LEMma 3.2. The right unit homomorphism is given by

$$
\eta_{\mathrm{R}}\left(\lambda_{k}\right)=\sum_{i=1}^{k}\left(\lambda_{i}-h_{i}\right) p_{i},
$$

where $p_{i} \in \mathbb{Z}\left[h_{1}, \ldots, h_{k-i}\right]$, and $p_{k}=1$.

Pr o of. Apply the conjugation $c$ to the standard formula

$$
\eta_{\mathrm{R}}\left(\lambda_{k}\right)=\sum_{i=0}^{k} \lambda_{i} t_{k-i}^{p^{i}}
$$

The resulting formula is

$$
\lambda_{k}=\sum_{i=0}^{k} \eta_{\mathrm{R}}\left(\lambda_{i}\right) h_{k-i}^{p^{i}} .
$$

Here the fact that $c\left(t_{j}\right)=h_{j}$ is used. A simple manipulation of terms yields

$$
\eta_{\mathrm{R}}\left(\lambda_{k}\right)=\left(\lambda_{k}-h_{k}\right)-\sum_{i=1}^{k-1} \eta_{\mathrm{R}}\left(\lambda_{i}\right) h_{k-i}^{p^{i}} .
$$

By induction, it may be assumed that $\eta_{\mathrm{R}}\left(\lambda_{i}\right)$ has the desired form for all $i<k$. Then it is evident that $\eta_{\mathrm{R}}\left(\lambda_{k}\right)$ also has the desired form.

Proof of Lemma 3.1. One may readily see from (30) that

$$
\mathcal{P}^{\Delta_{k}}\left(\lambda_{k}\right)=-1 \text {. }
$$

It is a bit more difficult to see that, when $j \neq k$,

$$
\mathcal{P}^{\Delta_{j}}\left(\lambda_{k}\right)=0 .
$$

This follows from (30) and the fact that $\left|p_{i}\right|=2\left(p^{k}-1\right)-2\left(p^{i}-1\right)=$ $2\left(p^{k}-p^{i}\right)$, which is never equal to $\left|h_{j}\right|=2\left(p^{j}-1\right)$ for any $j$. The general formula

$$
\left(\mathcal{P}^{\Delta}\right)^{E} \lambda^{F}=(-1)^{e(E)}(F ! /(F-E) !) \lambda^{F-E}
$$

now follows from the fact that the $\mathcal{P}^{\Delta_{j}}$ all commute with each other, and act as derivations.

Proof of Theorem 1.1. First we will show that the coefficient ring formula for $M_{\delta}$ gives rise to the $p$-typical curve indicated. Then we will show that the action on the coefficient ring is the one that is claimed. 
Suppose that

$$
M_{\delta}\left(\lambda_{k}\right)=\sum_{j=0}^{k} \chi\left(\delta_{k-j}\right) \lambda_{j} u^{p^{k}-p^{j}}
$$

Let

$$
\text { (38) } \begin{aligned}
\operatorname{mog} x & =\sum_{k \geq 0} M_{\delta}\left(\lambda_{k}\right) x^{p^{k}} \\
& =\sum_{k \geq 0} \sum_{j=0}^{k} \chi\left(\delta_{k-j}\right) \lambda_{j} u^{p^{k}-p^{j}} x^{p^{k}}=\sum_{n \geq 0} \sum_{j \geq 0} \chi\left(\delta_{n}\right) \lambda_{j} u^{p^{n+j}-p^{j}} x^{p^{n+j}} \\
& =\sum_{n \geq 0} \chi\left(\delta_{n}\right)\left(\sum_{j \geq 0} \lambda_{j}\left(u^{p^{n}-1} x^{p^{n}}\right)^{p^{j}}\right)=\sum_{n \geq 0} \chi\left(\delta_{n}\right) \log \left(u^{p^{n}-1} x^{p^{n}}\right)
\end{aligned}
$$

Then

$$
f_{\delta}^{-1}(x)=\exp (\operatorname{mog} x)=\sum_{n \geq 0}^{F}\left[\chi\left(\delta_{n}\right)\right]_{F} u^{p^{n}-1} x^{p^{n}} .
$$

For each $k \geq 0$ let $g_{k} \in H_{*}\left(\mathrm{BP} ; \mathbb{Z}_{(p)}\right)\left[h_{1}, \ldots, h_{k}\right]$ be the polynomial given by $g_{k}\left(h_{1}, \ldots, h_{k}\right)=\eta_{\mathrm{R}}\left(\lambda_{k}\right)$. If we let $c_{k} \in H_{*}\left(\mathrm{BP} ; \mathbb{Z}_{(p)}\right)\left[u, u^{-1}\right]$ be given by the formula $c_{k}(u)=g_{k}\left(\delta_{1} u^{p-1}, \delta_{2} u^{p^{2}-1}, \ldots, \delta_{k} u^{p^{k}-1}\right)$, then it follows by the definition of $M_{\delta}$ that

$$
M_{\delta}\left(\lambda_{k}\right)=c_{k}(u) .
$$

To complete the proof of the theorem, we will show by induction that

$$
c_{k}(u)=\sum_{j=0}^{k} \chi\left(\delta_{k-j}\right) \lambda_{j} u^{p^{k}-p^{j}} .
$$

In the case $k=1$, we see that

$$
c_{1}(u)=\lambda_{1}-\delta_{1} u^{p-1} .
$$

Hence the result is true in the initial case.

For general $k$, assume the result holds for all $c_{i}(u)$, where $i<k$. Then using (33) we find

$$
\begin{aligned}
c_{k}(u) & =\left(\lambda_{k}-\delta_{k} u^{p^{k}-1}\right)-\sum_{i=1}^{k-1} c_{i}(u)\left(\delta_{k-i} u^{p^{k-i}-1}\right)^{p^{i}} \\
& =\left(\lambda_{k}-\delta_{k} u^{p^{k}-1}\right)-\sum_{i=1}^{k-1}\left(\sum_{j=0}^{i} \chi\left(\delta_{i-j}\right) \lambda_{j} u^{p^{i}-p^{j}}\right) \delta_{k-i} u^{p^{k}-p^{i}} \\
& =\lambda_{k}-\sum_{i=0}^{k-1} \sum_{j=0}^{i} \delta_{k-i} \chi\left(\delta_{i-j}\right) \lambda_{j} u^{p^{k}-p^{j}}
\end{aligned}
$$




$$
\begin{aligned}
& =\lambda_{k}+\sum_{j=0}^{k-1}\left(-\sum_{i=j}^{k-1} \delta_{k-i} \chi\left(\delta_{i-j}\right)\right) \lambda_{j} u^{p^{k}-p^{j}} \\
& =\lambda_{k}+\sum_{j=0}^{k-1}\left(-\sum_{m=0}^{k-j-1} \delta_{k-j-m} \chi\left(\delta_{m}\right)\right) \lambda_{j} u^{p^{k}-p^{j}} \\
& =\lambda_{k}+\sum_{j=0}^{k-1} \chi\left(\delta_{k-j}\right) \lambda_{j} u^{p^{k}-p^{j}} \\
& =\sum_{j=0}^{k} \chi\left(\delta_{k-j}\right) \lambda_{j} u^{p^{k}-p^{j}} .
\end{aligned}
$$

Theorem 1.1 has some interesting special cases, corresponding to when $\delta=(1,0,0, \ldots)$, and when $\delta=(1,1,1, \ldots)$.

COROLlaRY 3.3. The multiplicative operation $\sum_{m \geq 0} u^{m(p-1)} \mathcal{P}^{m}$ corresponds to the p-typical curve

$$
f^{-1}(x)=\sum_{n \geq 0}^{F}\left[(-1)^{n}\right]_{F} u^{p^{n}-1} x^{p^{n}} .
$$

The multiplicative operation $\sum_{E} u^{|E|} \mathcal{P}^{E}$ corresponds to the p-typical curve

$$
g^{-1}(x)=x-{ }_{F} u^{p-1} x^{p} .
$$

Proof of Theorem 1.3. Because $M_{\delta}$ is a multiplicative operation, it is determined by its value on $\lambda_{k}$ for each $k \geq 0$. In particular,

$$
M_{\delta}\left(\lambda_{k}\right)=\sum_{j=0}^{k} \chi\left(\delta_{k-j}\right) \lambda_{j} u^{p^{k}-p^{j}} .
$$

The $\mathcal{P}^{\Delta_{i}}$ operate as derivations. Hence one may construct multiplicative operations from the $\mathcal{P}^{\Delta_{i}}$ by using the series

$$
\operatorname{Exp}(x)=\sum_{n \geq 0} \frac{x^{n}}{n !}
$$

where $x$ is taken to be $a_{k} u^{p^{k}-1+\left|a_{k}\right|} \mathcal{P}^{\Delta_{k}}$ for some $a_{k}$ in $\mathrm{BP}^{*} \otimes \mathbb{Q}$. The formula for $\mathcal{P}^{\Delta_{i}}\left(\lambda_{k}\right)$ given by Lemma 3.1 allows one to compute

$$
\operatorname{Exp}\left(a_{k} u^{p^{k}-1+\left|a_{k}\right|} \mathcal{P}^{\Delta_{k}}\right)\left(\lambda_{k}\right)=\lambda_{k}-a_{k} u^{p^{k}-1+\left|a_{k}\right|} .
$$

If we set

$$
a_{k}=-\sum_{j=0}^{k-1} \chi\left(\delta_{k-j}\right) \lambda_{j} u^{p^{k}-p^{j}}
$$


then

$$
\begin{aligned}
M_{\delta}\left(\lambda_{k}\right) & =\operatorname{Exp}\left(a_{k} u^{p^{k}-1+\left|a_{k}\right|} \mathcal{P}^{\Delta_{k}}\right)\left(\lambda_{k}\right) \\
& =\operatorname{Exp}\left(-\sum_{j=0}^{k-1} \chi\left(\delta_{k-j}\right) \lambda_{j} u^{p^{k}-p^{j}} \mathcal{P}^{\Delta_{k}}\right)\left(\lambda_{k}\right) .
\end{aligned}
$$

However, for $i \neq k$,

$$
\operatorname{Exp}\left(-\sum_{j=0}^{k-1} \chi\left(\delta_{k-j}\right) \lambda_{j} u^{p^{k}-p^{j}} \mathcal{P}^{\Delta_{k}}\right)\left(\lambda_{i}\right)=\lambda_{i},
$$

whereas

$$
M_{\delta}\left(\lambda_{i}\right)=\sum_{j=0}^{i} \chi\left(\delta_{i-j}\right) \lambda_{j} u^{p^{i}-p^{j}}=\lambda_{i}+\sum_{j=0}^{i-1} \chi\left(\delta_{i-j}\right) \lambda_{j} u^{p^{i}-p^{j}} .
$$

In order to compensate for this, we note that the product

$$
\prod_{k \geq 1} \operatorname{Exp}\left(-\sum_{j=0}^{k-1} \chi\left(\delta_{k-j}\right) \lambda_{j} u^{p^{k}-p^{j}} \mathcal{P}^{\Delta_{k}}\right)\left(\lambda_{i}\right)=M_{\delta}\left(\lambda_{i}\right)
$$

for every $i \geq 0$. However, one must take care to order this product correctly. The correct ordering is the one in which the $\operatorname{Exp}\left(a_{k} u^{p^{k}-1+\left|a_{k}\right|} \mathcal{P}^{\Delta_{k}}\right)$ for smaller values of $k$ are written to the right of the $\operatorname{Exp}\left(a_{k} u^{p^{k}-1+\left|a_{k}\right|} \mathcal{P}^{\Delta_{k}}\right)$ for larger values of $k$. The need for this ordering arises from the fact that the $\mathcal{P}^{\Delta_{k}}$ act non-trivially on $\lambda_{k}$. To complete the proof, the ordered product

$$
M_{\delta}=\prod_{k \geq 1} \operatorname{Exp}\left(-\sum_{j=0}^{k-1} \chi\left(\delta_{k-j}\right) \lambda_{j} u^{p^{k}-p^{j}} \mathcal{P}^{\Delta_{k}}\right)
$$

may be rewritten as the Exp of an ordered sum:

$$
\begin{aligned}
M_{\delta} & =\operatorname{Exp}\left(\sum_{k \geq 1} \sum_{j=0}^{k-1}-\chi\left(\delta_{k-j}\right) \lambda_{j} u^{p^{k}-p^{j}} \mathcal{P}^{\Delta_{k}}\right) \\
& =\operatorname{Exp}\left(-\sum_{0 \leq j<k} \chi\left(\delta_{k-j}\right) \lambda_{j} u^{p^{k}-p^{j}} \mathcal{P}^{\Delta_{k}}\right),
\end{aligned}
$$

where the ordering from left to right is decreasing with respect to $k$.

4. The Hopf invariant. In this section, a short proof of the wellknown Hopf invariant one theorem [1], [8] is given which uses the exponential relations of Theorem 1.3. An interesting point to note is that this proof works simultaneously for all primes. 
TheOREM 4.1. If $X$ is a p-cell complex, and the mod $p$ cohomology of $X$ is a truncated polynomial algebra,

$$
H^{*}(X ; \mathbb{Z} / p) \cong \frac{\mathbb{Z} / p[x]}{\left(x^{p+1}\right)},
$$

then the degree of $x$ is $2 p^{k}$, where either $k=0$ or $p^{k}-p^{k-1} \leq k$. Hence $k=0$ if $p$ is odd, and $k \leq 2$ if $p=2$.

Pr o of. It is well known that the Adem relations in the mod $p$ Steenrod algebra force the degree of $x$ to be twice a power of $p$. The main body of the proof here is to restrict the possible values of $k$. For the duration of this proof, let $\delta=(1,1,1, \ldots)$. We use the relation

$$
M_{\delta}=\operatorname{Exp}\left(\sum_{k \geq 1} \lambda_{k-1} u^{p^{k}-p^{k-1}} \mathcal{P}^{\Delta_{k}}\right) .
$$

Formula (57) may be simplified to

$$
M_{\delta}=\operatorname{Exp}\left(u^{p-1} \mathcal{P}^{1}\right) \bmod \left(\lambda_{1}, \lambda_{2}, \ldots\right) .
$$

If we write

$$
M_{\delta}=\sum_{n \geq 0} u^{n} M_{\delta, n}
$$

then (58) may be simplified even further to

$$
\left(\mathcal{P}^{1}\right)^{p^{k}}=\left(p^{k}\right) ! M_{\delta, p^{k}(p-1)} \bmod \left(\lambda_{1}, \lambda_{2}, \ldots\right) .
$$

Because $H^{*}\left(X ; \mathbb{Z}_{(p)}\right)$ is torsion free,

$$
\mathrm{BP}^{*}(X) \cong \mathrm{BP}^{*}[\widetilde{x}] /\left(\widetilde{x}^{p+1}\right),
$$

where the Thom map BP $\rightarrow H \mathbb{Z} / p$ takes $\widetilde{x}$ to $x$. Applying both sides of (60) to $\widetilde{x}$ yields

$$
\begin{aligned}
\left(\mathcal{P}^{1}\right)^{p^{k}} \widetilde{x} & =\left(p^{k}\right) ! M_{\delta, p^{k}(p-1)} \widetilde{x} \bmod \left(\lambda_{1}, \lambda_{2}, \ldots\right) \\
& =\left(p^{k}\right) ! \mathcal{P}^{p^{k}} \widetilde{x} \bmod \left(p\left(p^{k}\right) !, \lambda_{1}, \lambda_{2}, \ldots\right) \\
& =\left(p^{k}\right) ! \widetilde{x}^{p} \bmod \left(p\left(p^{k}\right) !, \lambda_{1}, \lambda_{2}, \ldots\right) .
\end{aligned}
$$

Now it is not difficult to see that, for degree reasons, there is a number $c \in \mathbb{Z}_{(p)}$ for which

$$
\mathcal{P}^{1} \widetilde{x}=c \lambda_{1}^{p^{k}-1} \widetilde{x}^{p} \bmod \left(\lambda_{2}, \lambda_{3}, \ldots\right) .
$$

Applying $\left(\mathcal{P}^{1}\right)^{p^{k}-1}$ to both sides of (63) and using the fact that $\mathcal{P}^{1}$ is a derivation yields

$$
\left(\mathcal{P}^{1}\right)^{p^{k}} \widetilde{x}=c(-1)^{p^{k}-1}\left(p^{k}-1\right) ! \widetilde{x}^{p} \bmod \left(\lambda_{1}, \lambda_{2}, \lambda_{3}, \ldots\right) .
$$


If we now combine (62), (64) and (63), we may conclude that

$$
c=p^{k} \bmod p^{k+1}, \quad c \lambda_{1}^{p^{k}-1} \in \mathrm{BP}_{*} \bmod \left(\lambda_{2}, \lambda_{3}, \ldots\right) .
$$

If $v_{i} \in \mathrm{BP}_{*}$ is the Hazewinkel generator, then

$$
v_{i}=p^{p^{i-1}} \lambda_{1}^{\left(p^{i}-1\right) /(p-1)} \bmod \left(\lambda_{2}, \lambda_{3}, \ldots\right) .
$$

From the second part of (65), and from (66) one may conclude that when $k>0, c$ must be divisible by $\left(p^{p^{k-1}}\right)^{p-1}$. Combining this with the first part of (65) implies $p^{k-1}(p-1) \leq k$, or $p^{k}-p^{k-1} \leq k$, the desired conclusion.

\section{References}

[1] J. F. Adams, On the non-existence of elements of Hopf invariant one, Ann. of Math. 72 (1960), 20-104.

[2] S. Araki, Multiplicative operations in BP cohomology, Osaka J. Math. 12 (1975), 343-356.

[3] J. M. Boardman, The eightfold way to BP operations, Canad. Math. Soc. Proc. 2 (1982), 187-226.

[4] J. R. Hubbuck, Generalized cohomology operations and H-spaces of low rank, Trans. Amer. Math. Soc. 141 (1969), 335-360.

[5] R. Kane, Rational BP operations and the Chern character, Math. Proc. Cambridge Philos. Soc. 84 (1978), 65-72.

[6] -, Brown-Peterson operations and Steenrod modules, Quart. J. Math. Oxford 30 (1979), 455-467.

[7] P. S. Landweber, Homological properties of comodules over $M U_{*} M U$ and $B P_{*} B P$, Amer. J. Math. 98 (1976), 591-610.

[8] A. L. Liulevicius, The factorization of cyclic reduced powers by secondary cohomology operations, Mem. Amer. Math. Soc. 42 (1962).

[9] J. W. Milnor, The Steenrod algebra and its dual, Ann. of Math. 67 (1958), 150-171.

[10] D. C. Ravenel, Complex Cobordism and Stable Homotopy Groups of Spheres, Academic Press, 1986.

Department of Mathematics and Statistics

Western Michigan University

Kalamazoo, Michigan 49008-5152

U.S.A.

E-mail: slack@math-stat.wmich.edu

Received 20 July 1998;

in revised form 27 October 1998 